\begin{tabular}{|c|c|c|}
\hline Beitr. Ent. & Berlin & ISSN 0005-805X \\
\hline $\mathbf{5 0}(2000) 2$ & S. $265-269$ & 02.10 .2000 \\
\hline
\end{tabular}

\title{
Deliphrosoma lothari sp. n. della Turchia N-orientale
}

\section{(Coleoptera, Staphylinidae) ${ }^{1}$}

\section{A 5 figuras}

ARNALDO BORDONI

\section{Summary}

Deliphrosoma lothari sp. n. from Kopdagi gecidi in NE-Turkey is described. It is closely related to D. mandli (SCHEERPELTZ), but differing in the morphology of the body and of the aedeagus. The new species is also compared with $D$. kopdagense BORDONI from the same locality and with $D$. freudeorum ZERCHE and D. turcicum ZERCHE from East Anatolia. A new distribution map is given for all the species known from Anatolia.

\section{Zusammenfassung}

Deliphrosoma lothari sp. $\mathrm{n}$. vom Kopdagi gecidi in der Nordost-Türkei wird beschrieben. Die neue Art ist eng verwandt mit $D$. mandli (SCHEERPELTZ), unterscheidet sich aber durch ektoskelettale Merkmale und durch den Bau des Aedoeagus. Die neue Art wird auch mit $D$. kopdagense BORDONI von der gleichen Lokalität und mit $D$. freudeorum ZERCHE und $D$. turcicum ZERCHE aus Ost-Anatolien verglichen. Für die bisher aus Anatolien bekannten Arten wird eine neue Verbreitungskarte gegeben.

In un contributo precedente (BORDONI 1999) ho descritto una specie di Deliphrosoma (D. kopdagense) dei Monti Kopdagi presso Erzurúm, raccolta sotto pietre a 2300-2400 m s.l.m. Uno degli esemplari era stato trovato da A. RIEDEL a $2300 \mathrm{~m}$ assieme ad altri due che appartengono ad una specie diversa ed inedita che quindi convive con $D$. kopdagense BORDONI.

In questa sede descrivo questa specie come:

\section{Deliphrosoma lothari sp. n.}

\section{Serie tipica}

Holotypus ( $\left.\sigma^{\pi}\right)$ : Türkei, Anatolien, Erzurum, Kop-Gec, 19.5.1989, 2300 m, leg. A. Riedel (SMNS); paratypus $\left(0^{*}\right)$ : stessi dati $(\mathrm{CB}){ }^{2}$

${ }^{1} 118^{\circ}$ contributo alla conoscenza degli Staphylinidae.

${ }^{2}$ Sigle adottate: SMNS (Staatliches Museum für Naturkunde, Stuttgart); CB (collezione BORDONI, Firenze). 


\section{Diagnosi}

Un Deliphrosoma di $3,95 \mathrm{~mm}$ di lunghezza che convive con $D$. kopdagense Bordoni in Anatolia e dal quale differisce per il corpo più piccolo, per la diversa forma di capo-pronotoelitre, per gli occhi assai meno sporgenti, per il pronoto con angoli posteriori non sporgenti e subacuti, per gli ocelli del capo più distanziati tra loro, per la punteggiatura e per l'organo copulatore maschile più piccolo e con lobi laterali diversamente conformati.

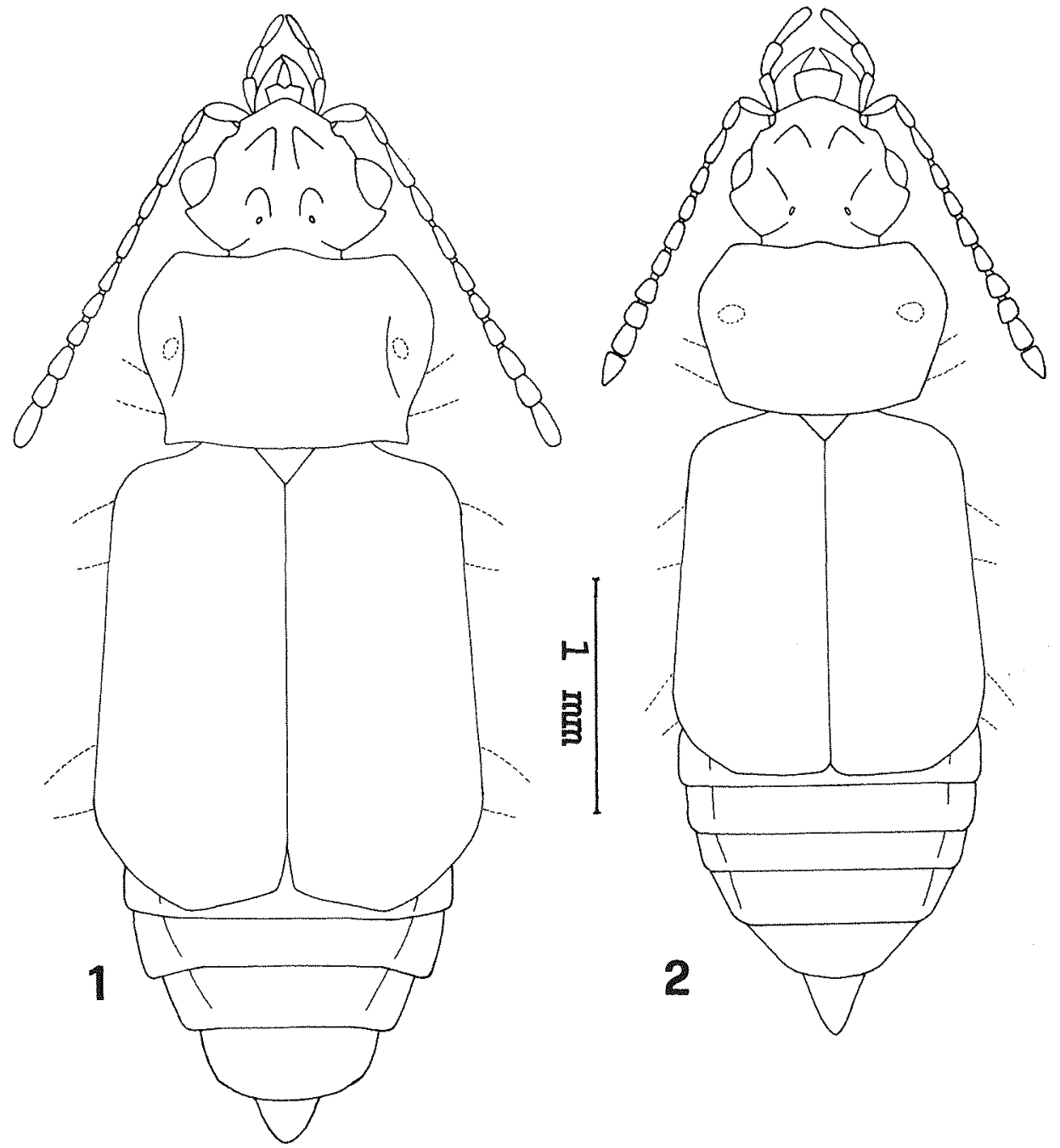

Figg. 1-2: Habitus: 1. Deliphrosoma kopdagense BORDONI - 2. D. lothari sp. n.

\section{Descrizione}

Corpo (fig. 2) lungo circa $3,95 \mathrm{~mm}$; lunghezza dal margine anteriore del capo a quello posteriore delle elitre di $2,83 \mathrm{~mm}$ (il paratipo misura rispettivamente $4 \mathrm{~mm}$ e $2,76 \mathrm{~mm}$ ). Colorazione un poco variabile, bruna con capo più scuro ed elitre più chiare. 
Capo appena più largo che lungo e quindi poco dilatato, con occhi piccoli e assai poco sporgenti, nettamente più corti delle tempie. Superficie del capo microstriata in maglie trasversali irregolari, quasi longitudinali sul disco, poligonali e fitte dietro i tubercoli antennali. Antenne più o meno come nelle altre specie.

Pronoto ad angoli anteriori e posteriori arrotondati, con fossetta laterale profonda. Superficie con tracce di microstriatura trasversale in avanti ed in addietro, quindi per gran parte lucida e liscia, con punteggiatura netta e fitta.

Elitre appena dilatate in addietro, ad angoli posteriori largamente arrotondati. Superficie lucida e liscia con punteggiatura netta, profonda, disposta in numerosissime serie molto accostate tra loro. Addome con finissima e fitta microstriatura più o meno poligonale e con punteggiatura molto fine e molto rada.

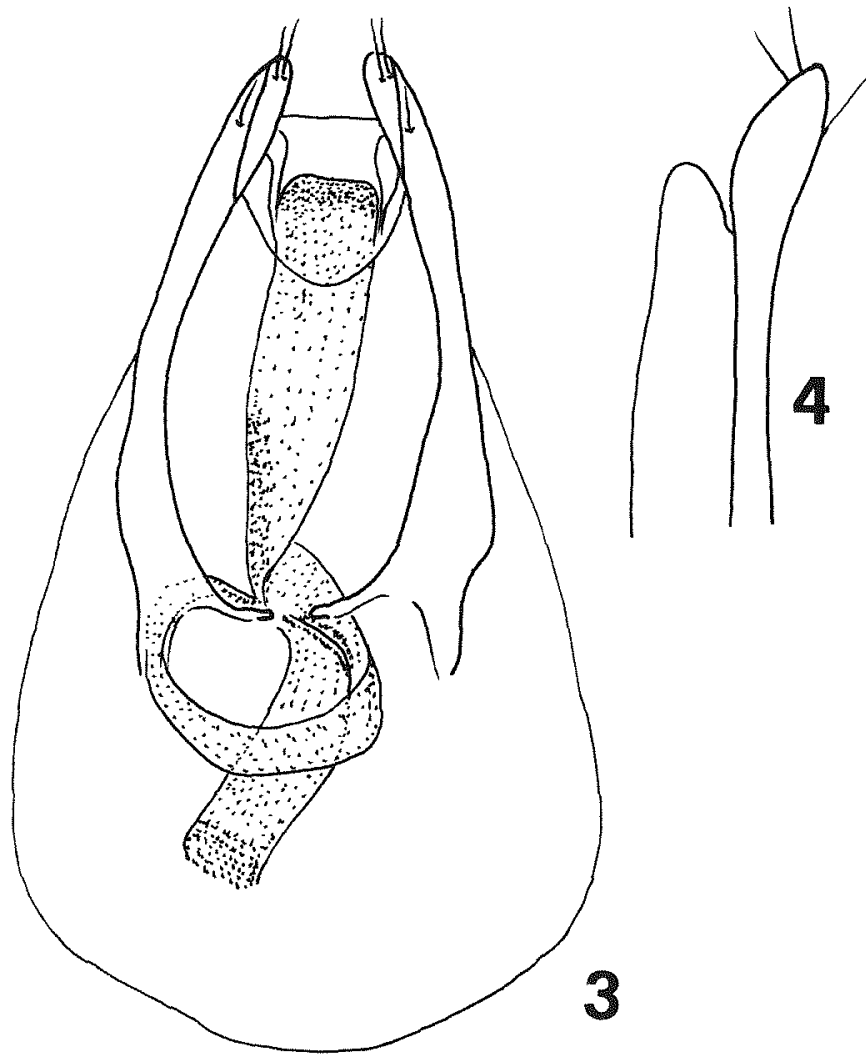

Figg. 3-4: Deliphrosoma lothari sp. n.: 3. organo copulatore maschile in visione ventrale - 4. apice dei lobi laterali in visione laterale.

Organo copulatore (fig. 3) largo, con lobi laterali discretamente più lunghi del lobulo mediano, ad apice piuttosto ristretto e provvisto di tre setole; sacco interno piuttosto largo e ricoperto di finissime scagliette, più addensate nelle porzioni distale, mediana e prossimale. I lobi laterali, in visione laterale, hanno forma caratteristica (fig. 4).

\section{Derivatio nominis}

Dedico con piacere questa nuova specie al collega ed amico dr. LOTHAR ZERCHE del Deutsches Entomologisches Institut di Eberswalde, specialista del gruppo. 


\section{Osservazioni}

Questa specie è stata raccolta assieme a $D$. kopdagense Bordoni per cui mi sembra opportuno confrontarla con questa (figs. 1-2): la nuova specie risulta sensibilmente più piccola, con la lunghezza di capo-pronoto-elitre che varia da 2,76 a 2,83 mm mentre in kopdagense varia da 3,20 a 3,50; la forma del capo è differente, in quanto meno subtriangolare, meno dilatato, con occhi più piccoli e assai meno sporgenti; gli ocelli sono molto più distanziati tra loro; il pronoto è più piccolo ed ha angoli posteriori smussati e quindi non sporgenti e subacuti; le elitre sono più piccole, proporzionalmente molto più corte, meno dilatate in addietro; la punteggiatura del corpo è più rada e la superficie è più lucida.

L'organo copulatore è ben più piccolo in lothari sp. n. $(0,8 \mathrm{~mm})$ che in kopdagense BORDONI $(0,9 \mathrm{~mm})$ ed ha lobi laterali diversamente conformati; essi sono meno dilatati all'apice anche in visione laterale. Il sacco interno è simile nella struttura generale ma più largo e ricoperto da scagliette più fitte.

Rispetto a $D$. freudeorum ZERCHE, la nuova specie è molto più piccola ed ha organo copulatore diversamente conformato, con lobi laterali provvisti di due sole setole apicali. Anche D. turcicum ZERCHE ha corpo più grande e lobi laterali con due sole setole apicali. Assieme a $D$. weiratheri (SCHEERPELTZ) queste due entità formano un gruppo di specie affini. D. lothari sp. $\mathrm{n}$. sistematicamente si avvicina piuttosto a $D$. mandli (SCHEERPELTZ) da cui si differenzia per la maggiore lucentezza dei tegumenti, per la microstriatura assai meno evidente, per il $5^{\circ}$ antennomero un poco più lungo del $4^{\circ}$ e per i caratteri sessuali: organo copulatore un poco più grande, con lobi laterali meno dilatati all'apice anche in visione laterale e con sacco interno più corto e più largo.
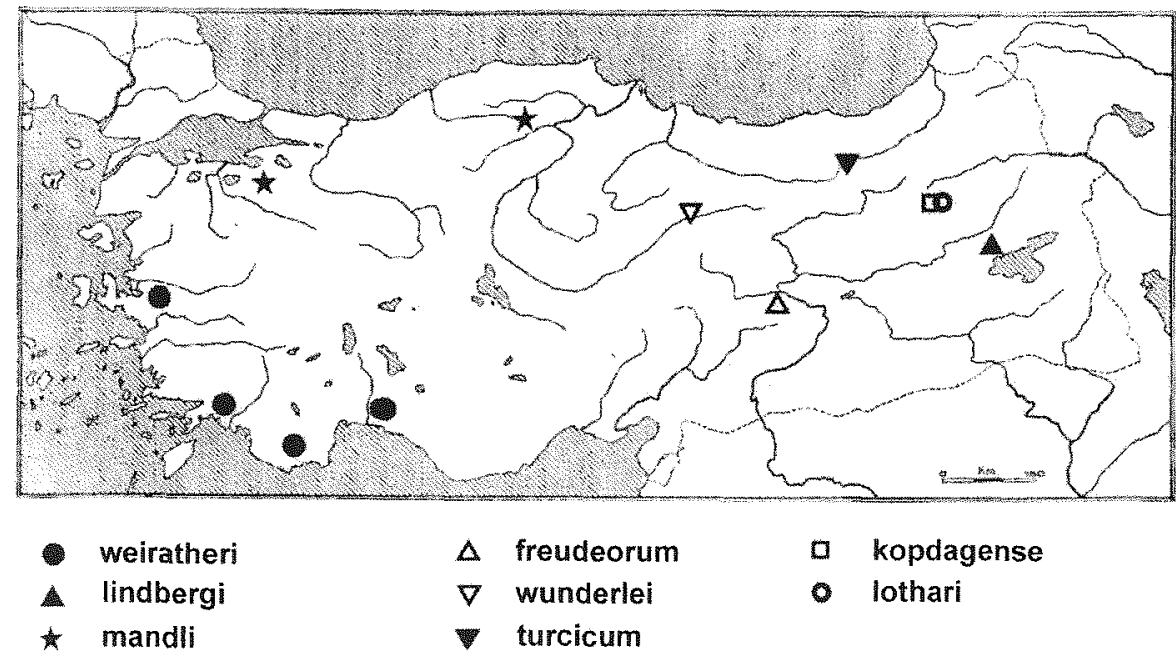

$\Delta$ freudeorum

a kopdagense

$\nabla$ wunderlei

- lothari

mandli

turcicum

Fig. 5: Distribuzione del genere Deliphrosoma REITTER in Turchia.

Le specie di Deliphrosoma attualmente note della Turchia sono otto (fig. 5). Alcune di queste sono state descritte alcuni decenni orsono (cfr. SCHEERPELTZ 1937a, 1937b, 1958): weiratheri (SCHEERPElTZ) del Tauro di Lidia, Panfilia e Caria (BORDONI 1984), mandli (SCHEERPELTZ) della Bitinia (nota anche di Bulgaria), lindbergi (SCHEERPELTZ) della Grotta Soltan Séit presso Ahlat a NW del Lago Van che è situato oltre i $1600 \mathrm{~m}$ nell'Armenia turca (di cui non è noto il maschio); queste specie sono state ristudiate recentemente (ZERCHE 1991a, 1991b; BORDONI 1984, 1999). Alcune altre sono state descritte negli ultimi anni: freudeorum ZERCHE dei Monti 
Nemrat presso Malatya nella Cappadocia meridionale (ZERCHE 1996), turcicum ZERCHE di una località imprecisata presso Gümüshane nel Ponto ma presumibilmente sulle montagne più continentali tra quelle che la circondano, per le affinità con specie anatoliche (ZERCHE 1997), wunderlei ZERCHE dei Kizildag presso Sivas nella Cappadocia N-occidentale (ZERCHE 1998), kopdagense BORDONI dei Monti Kopdagi presso Erzurum nella Cappadocia settentrionale (BORDONI 1999) e la nuova specie qui descritta della medesima località.

I Deliphrosoma sono Stafilinidi propri delle alte quote e, a parte alcuni casi, le specie hanno areali molto ristretti. In Turchia solo weiratheri e mandli hanno un areale più esteso, il primo essendo conosciuto dell'Anatolia occidentale e S-occidentale ed il secondo degli Uludagh e degli Ilgasdagh nella Turchia settentrionale e dei Monti Vitoscha e delle montagne dei Balcani centrali in Bulgaria (cfr. anche ZERCHE 1991a, 1998, 1999). Le altre specie attualmente conosciute della Turchia sono endemiti montani; non è improbabile quindi che le principali catene montuose della Turchia ospitino specie differenti. I dati non sono numerosi tuttavia i recenti studi stanno evidenziando che questo genere è ben rappresentato nella regione.

\section{Ringraziamenti}

Desidero ringraziare i colleghi dr. A. RIEDEL (Friedberg) per le informazioni fornitemi, dr. W. SCHAWALLER (Stuttgart) per avermi inviato in studio questi esemplari, dr. L. ZERCHE (Eberswalde) per la collaborazione e per il controllo della specie.

\section{Bibliografia}

BORDONi A. 1984: Note su alcuni Stafilinidi del Libano (Coleoptera). - Fragm. Entomol., Roma 17 (2): 331-345.

Bordoni A. 1999: Una nuova specie di Deliphrosoma della Turchia (Coleoptera, Staphylinidae). Fragm. Entomol., Roma 31 (2): 195-203.

SCHEERPELTZ, O. 1937a: Bestimmungsschlüssel der paläartischen Arten der Gattung Arpedium ER., Untergattung Deliphrosoma REITT., nebst Beschreibung einer neuen Art (Col. Staphylinidae). - Kol. Rundsch., Wien 23: 229-235.

SCHEERPELTZ O. 1937b: Wissenschaftliche Ergebnisse einer von Herrn Hofrat F. SCHUBERT, seinen Sohne Herrn cand. phil. F. SCHUBERT und Herrn Prof. Ing. K. MANDL im Sommer 1935 (1936) nach Bulgarien unternommenen Studienreise. Coleoptera: I. Staphylinidae. - Mitt. Königl. Naturwiss. Inst. Sofia-Bulgarien 10: 185-246.

SCHEERPELTZ, O. 1958: Wissenschaftliche Ergebnisse der von Herrn Dr. K. LINDBERG, Lund, im Jahre 1956 nach der Türkei und Armenien unternommenen Reise. Coleoptera Staphylinidae. - Entomol. Tidskrift, Lund 78 (1957): 3-37.

ZERCHE L. 1991a: Beitrag zur Taxonomie und Verbreitung der Gattung Deliphrosoma ReITTER, 1909 (Coleoptera, Staphylinidae, Omaliinae). - Beitr. Ent., Berlin 41 (2): 313-332.

ZERCHE L. 1991b: Zur Taxonomie und Verbreitung der Gattung Deliphrosoma REITTER (Staphylinidae, Omalinae). - Verh. Westd. Entom. Tag 1990, Düsseldorf: 147-153.

ZERCHE L. 1996: Deliphrosoma freudeorum spec. nov. aus der Osttürkei (Insecta: Coleoptera: Staphylinidae: Omaliinae). - Reichenbachia Mus. Tierkd. Dresden 31 (31): 175-177.

ZERCHE L. 1997: Eine neue Art der Gattung Deliphrosoma REITTER aus der Nordost-Türkei und Ergänzungen zur Verbreitung der Gattung (Insecta: Coleoptera: Staphylinidae: Omaliinae). - Reichenbachia Mus. Tierkd. Dresden 32 (9): 49-52.

ZERCHE L. 1998: Neue Deliphrosoma-Arten aus Bulgarien und aus der Türkei und neue Unterarten von Deliphrosoma prolongatum (ROTTENBERG) aus Bulgarien (Insecta: Coleoptera: Staphylinidae: Omaliinae). - Reichenbachia Mus. Tierkd. Dresden 32 (36): 245- 255.

ZERCHE, L. 1999: Biodiversitätsforschung in Europa am Beispiel bulgarischer Hochgebirge (Coleoptera: Staphylinidae, Leiodidae). - Cour. Forsch.-Inst. Senckenberg, Frankfurt a. M. 215: 233-238.

\section{Indirizzo dell'autore:}

dr. ARNAldo BORDONI, c/o Museo di Storia Naturale dell'Università degli Studi di Firenze,

Sezione Zoologica "La Specola", via Romana 17, I-50125 Firenze, Italia 